\title{
RESEARCH
}

Open Access

\section{Linoleic acid improves assembly of the CII subunit and CIII2/CIV complex of the mitochondrial oxidative phosphorylation system in heart failure}

Satoshi Maekawa', Shingo Takada ${ }^{1,2,3^{*}}$ (D. Hideo Nambu' ${ }^{1}$, Takaaki Furihata ${ }^{1}$, Naoya Kakutani ${ }^{1,4}$, Daiki Setoyama ${ }^{5}$, Yasushi Ueyanagi ${ }^{5,6}$, Dongchon Kang ${ }^{5,6}$, Hisataka Sabe ${ }^{2 \dagger}$ and Shintaro Kinugawa ${ }^{1+}$

\begin{abstract}
Background: Linoleic acid is the major fatty acid moiety of cardiolipin, which is central to the assembly of components involved in mitochondrial oxidative phosphorylation (OXPHOS). Although linoleic acid is an essential nutrient, its excess intake is harmful to health. On the other hand, linoleic acid has been shown to prevent the reduction in cardiolipin content and to improve mitochondrial function in aged rats with spontaneous hypertensive heart failure (HF). In this study, we found that lower dietary intake of linoleic acid in HF patients statistically correlates with greater severity of $\mathrm{HF}$, and we investigated the mechanisms therein involved.

Methods: HF patients, who were classified as New York Heart Association (NYHA) functional class I $(n=45)$, II $(n=$ 93), and III ( $n=15)$, were analyzed regarding their dietary intakes of different fatty acids during the one month prior to the study. Then, using a mouse model of HF, we confirmed reduced cardiolipin levels in their cardiac myocytes, and then analyzed the mechanisms by which dietary supplementation of linoleic acid improves cardiac malfunction of mitochondria.

Results: The dietary intake of linoleic acid was significantly lower in NYHA III patients, as compared to NYHA II patients. In HF model mice, both Cl-based and Cll-based OXPHOS activities were affected together with reduced cardiolipin levels. Silencing of CRLS1, which encodes cardiolipin synthetase, in cultured cardiomyocytes phenocopied these events. Feeding HF mice with linoleic acid improved both Cl-based and Cll-based respiration as well as left ventricular function, together with an increase in cardiolipin levels. However, although assembly of the respirasome (i.e., $\mathrm{Cl} / \mathrm{CIII} / 2 / \mathrm{CIV}$ complex), as well as assembly of $\mathrm{Cll}$ subunits and the $\mathrm{CIII} / \mathrm{CIV}$ complex statistically correlated with cardiolipin levels in cultured cardiomyocytes, respirasome assembly was not notably restored by dietary linoleic acid in HF mice. Therefore, although linoleic acid may significantly improve both Cl-based and Cllbased respiration of cardiomyocytes, respirasomes impaired by HF were not easily repaired by the dietary intake of linoleic acid.
\end{abstract}

Conclusions: Dietary supplement of linoleic acid is beneficial for improving cardiac malfunction in HF, but is unable to completely cure HF.

Keywords: Supercomplex, Myocardial infarction, Cardiolipin, Succinate dehydrogenase, Respirasome

\footnotetext{
* Correspondence: s-takada@hotmail.co.jp

${ }^{+}$Hisataka Sabe and Shintaro Kinugawa contributed equally to this work

'Department of Cardiovascular Medicine, Hokkaido University Graduate

School of Medicine, Kita-15, Nishi-7, Kita-ku, Sapporo 060-8638, Japan

${ }^{2}$ Department of Molecular Biology, Hokkaido University Graduate School of

Medicine, Sapporo, Japan

Full list of author information is available at the end of the article
}

(c) The Author(s). 2019 Open Access This article is distributed under the terms of the Creative Commons Attribution 4.0 International License (http://creativecommons.org/licenses/by/4.0/), which permits unrestricted use, distribution, and

reproduction in any medium, provided you give appropriate credit to the original author(s) and the source, provide a link to the Creative Commons license, and indicate if changes were made. The Creative Commons Public Domain Dedication waiver (http://creativecommons.org/publicdomain/zero/1.0/) applies to the data made available in this article, unless otherwise stated. 


\section{Background}

The prevalence of heart failure (HF) has increased globally, particularly in developed countries in recent decades [1]. Advances in our understanding of the underlying pathophysiologic mechanisms have given rise to new therapies for treating the increasing population with HF. Nonetheless, morbidity and mortality associated with HF and its financial implications remains a large concern. Thus, novel therapies that can improve the natural history of HF patients are urgently needed [2]. Likewise, what actually constitutes a 'balanced diet' that can prevent as well as treat HF should be reconsidered, together with identification of the molecular mechanisms therein involved.

An individual's daily diet can affect the severity of HF [3]. However, only fluid and salt restriction are recommended regarding diet therapy for HF patients. Fats, which are essential nutrients, are absorbed by the body and subsequently decomposed into fatty acids, which are components of energy substrates and plasma membranes (i.e., cells, nuclei, and mitochondria) $[4,5]$. Linoleic acid (18:2), an essential fatty acid, is a major component of cardiolipin, which is a specific mitochondrial phospholipid. Mulligan et al. reported that moderate consumption of linoleic acid in aged spontaneous hypertensive HF rats $\left(\mathrm{Mcc}^{\mathrm{facp}-/-}\right)$ prevented the reduction of cardiolipin content and the exacerbation of mitochondrial function [6], but its mechanism remains unknown.

Structural studies have shown that cardiolipin is important for stabilization (i.e., the assembly of complex subunits) of mitochondrial oxidative phosphorylation (OXPHOS) complex I (CI) in sheep, [7] complex II (CII) in $S$. cerevisiae (in the absence of CI), [8] complex III (CIII) in cows, [9] and complex IV (CIV) in S. cerevisiae [10]. Although a decrease in cardiolipin levels promotes the disassembly of these mitochondrial OXPHOS complexes, it has not yet been clearly demonstrated whether dietary intake of linoleic acid can restore the integrity of these complexes as well as cardiac dysfunction.

We here found that HF patients with greater pathological severity statistically take lower amounts of dietary linoleic acid compared with HF patients with lower severity. Consistent with a previous report of an animal experiment, [6] we then found that linoleic acid can improve cardiolipin levels that were otherwise reduced in cardiac mitochondria of $\mathrm{HF}$ model mice. Nevertheless, our study also suggests that linoleic acid supplementation cannot fully restore the mitochondrial respirasome in HF mice. Together with the molecular bases by which linoleic acid improves the OXPHOS activity of cardiac mitochondria, as well as left ventricular function, our study suggests that insufficient intake of linoleic acid might be associated with $\mathrm{HF}$.

\section{Methods}

\section{Patients with HF}

A multicenter and prospective cohort study investigating the effect of malnutritional status on clinical outcomes in patients with chronic HF was performed. All the enrolled patients treated at 10 institutes ((1) Department of Nutritional Management Center, Hokkaido University Hospital; (2) Department of Cardiology, Kitami Red Cross Hospital; (3) Department of Molecular Medicine and Therapeutics, Tottori University Faculty of Medicine; (4) Department of Cardiovascular Medicine, Kushiro City General Hospital; (5) Department of Cardiology, Keiwakai Ebetsu Hospital; (6) Division of Cardiology, Cardiovascular and Aortic Center of Saiseikai Fukuoka General Hospital; (7) Hikone Municipal Hospital; (8) Otaru Kyokai Hospital; (9) National Hospital Organization Hospital, Hokkaido; (10) Hokkaido Social Work Association Obihiro Hospital) provided their written informed consent to participate in this study. The study was approved by the institutional ethics committee at each participating site and was conducted in accordance with the ethical principles described in the Declaration of Helsinki (2013 revised version).

Patient eligibility criteria were as follows: age $\geq 20$ years; one or more hospitalizations owing to worsening HF before enrollment; outpatients with stable New York Heart Association (NYHA) functional class I, II, or III for longer than 1 month since their prior hospitalization; and available written informed consent of the patient. Patients were excluded if they had nephrotic syndrome, liver cirrhosis, cancer, a history of gastrointestinal surgery within the previous 3 months, or poorly controlled diabetes (hemoglobin A1c $>7.0 \%$ ). Patients who were taking steroids, anticancer agents, or antidepressants were excluded because those drugs are known to affect appetite, dietary content, and body composition.

\section{Assessment of dietary intake of fatty acids}

We analyzed each patient's dietary intake pattern of several fatty acids (palmitate, stearate, oleate, linoleic acid, arachidonate, and docosahexaenoic acid (DHA) during the month preceding analysis, using a brief selfadministered diet history questionnaire (BDHQ), and compared the results among the three groups (patients of NYHA classes I, II, and III). The BDHQ is a 4-page structured questionnaire that calculates the consumption frequency of 58 selected food and beverage items based on the Standard Tables of Food Composition in Japan. The BDHQ has been validated to have very good correlation with a 150-item comprehensive diet history questionnaire [11]. The value of each item was energy-adjusted by density methods (g/1000 kcal) for each patient. The BDHQ was performed by two or more registered dietitians who were blinded to the patients' clinical findings." 


\section{Establishment of myocardial infarction mice}

Male C57BL/6 J mice (9-12-weeks old; CLEA Japan, Tokyo) were bred in a pathogen-free environment and housed in an animal room maintained at $23-25^{\circ} \mathrm{C}$ under controlled conditions on a 12-h light/dark cycle. Diet and water were provided ad libitum.

The HF post-myocardial infarction model was established by ligating the left coronary artery as described $[12,13]$. A sham surgery operation without ligation of the coronary artery was also performed as a control; the experiments were mainly performed 4 weeks after the surgery in the surviving control $(n=10)$ and HF $(n=10)$ mice. For each surgical procedure, the mice were anesthetized with an intraperitoneal injection (i.p.) of a mixture of $0.3 \mathrm{mg} / \mathrm{kg}$ of medetomidine (Dorbene ${ }^{\circ}$, Kyoritsuseiyaku, Tokyo), $4.0 \mathrm{mg} / \mathrm{kg}$ of midazolam (Dormicum ${ }^{\circ}$, Astellas Pharma, Tokyo), and $5.0 \mathrm{mg} / \mathrm{kg}$ of butorphanol (Vetorphale, Meiji Seika Kaisha, Tokyo). The adequacy level of anesthesia was monitored based on the disappearance of the pedal withdrawal reflex.

After 4 weeks, echocardiography was performed. The mice were then sacrificed, and the hearts were excised. Biochemical measurements, analysis of mitochondrial respiration, and immunoblotting including sodium dodecyl sulfate-polyacrylamide gel electrophoresis (SDSPAGE) or blue native (BN)-PAGE were performed.

\section{Linoleic acid administration to mice}

Once mice developed HF after their myocardial infarction surgery, they were divided into two groups; one group was fed a normal-diet (CE-2; CLEA Japan, $n=34$ ) and the other group was fed a linoleic acid-rich diet $(n=12)$ until their sacrifice 4 weeks after surgery. The linoleic-acid diet was prepared as described previously [14]. It contained the following ingredients in weight\%: 20 casein, 5 high-linoleate safflower oil, $13.2 \alpha$ cornstarch, 10 sucrose, 5 cellulose, 3.5 mineral mixture, 1 vitamin mixture, $0.3 \mathrm{~L}$-cysteine, 0.25 choline bitartrate, 0.0014 tert-butylhydroquinone, and 41.75 cornstarch. After 4 weeks, echocardiography was performed, the mice were sacrificed, and their hearts were excised. Biochemical measurements, evaluation of mitochondrial respiration, and immunoblotting, including SDS-PAGE and BN-PAGE were performed.

\section{Echocardiography}

Standard echocardiographic short-axis views were obtained at the levels of the papillary muscles under light anesthesia using tribromoethanol/amylene hydrate (avertin; 2.5\% wt/ vol, $8 \mu \mathrm{L} / g$ body weight, i.p.). The left ventricular (LV) enddiastolic diameter, LV end-systolic diameter, and LV fractional shortening from M-mode frames at a paper speed of $50 \mathrm{~mm} / \mathrm{sec}$ were measured $[12,15]$.

Table 1 Baseline patient characteristics

\begin{tabular}{|c|c|c|c|c|}
\hline & NYHA I & NYHA ॥ & NYHA III & $P$-value \\
\hline Number & 45 & 93 & 15 & \\
\hline \multicolumn{5}{|l|}{ Demographic factors } \\
\hline Age, yrs & $66 \pm 12$ & $68 \pm 13$ & $67 \pm 18$ & 0.734 \\
\hline Men, n (\%) & $34(76)$ & $73(71)$ & $5(33)$ & 0.010 \\
\hline Body mass index, $\mathrm{kg} / \mathrm{m}^{2}$ & $23.7 \pm 3.3$ & $23.7 \pm 5.6$ & $23.3 \pm 5.8$ & 0.685 \\
\hline \multicolumn{5}{|l|}{ Cause of heart failure, n (\%) } \\
\hline Ischemic heart disease & $16(36)$ & $23(25)$ & $2(13)$ & 0.264 \\
\hline Dilated cardiomyopathy & $14(31)$ & $5(5)$ & $7(47)$ & 0.393 \\
\hline Valvular heart disease & $11(24)$ & $6(6)$ & $6(40)$ & 0.396 \\
\hline Hypertensive heart disease & $12(27)$ & $27(29)$ & $4(27)$ & 0.965 \\
\hline Hypertrophic cardiomyopathy & $0(0)$ & $3(3)$ & $0(0)$ & 0.189 \\
\hline \multicolumn{5}{|l|}{ Medication, n (\%) } \\
\hline$\beta$-blocker & $34(76)$ & $84(90)$ & $12(80)$ & 0.403 \\
\hline ACE-I or ARB & $28(62)$ & $76(82)$ & $11(73)$ & 0.029 \\
\hline Diuretics & $34(76)$ & $72(77)$ & $14(93)$ & 0.311 \\
\hline \multicolumn{5}{|l|}{ Echocardiographic parameters } \\
\hline LVEDD, mm & $55 \pm 9$ & $57 \pm 12$ & $62 \pm 13$ & 0.089 \\
\hline LVEF, \% & $46 \pm 14$ & $45 \pm 16$ & $35 \pm 16$ & 0.057 \\
\hline
\end{tabular}

NYHA class, New York Heart Association class; ACE-I, angiotensin converting enzyme inhibitor; ARB, angiotensin II receptor blocker; LVEDD, left ventricular enddiastolic diameter; LVEF, left ventricular ejection fraction. Data are expressed as means \pm SD 
Preparation of isolated mitochondria from mouse heart Mitochondria were isolated from mouse hearts. The heart was quickly harvested, and mitochondria were isolated as described previously [16-18]. With regard to the LVs from HF mice, infarct areas were excluded, and only non-infarct areas were used for the experiments. Briefly, heart tissues were minced on ice and then incubated for $2 \mathrm{~min}$ in mitochondrial isolation buffer $(100 \mathrm{mmol} / \mathrm{L}$ sucrose, $100 \mathrm{mmol} /$ $\mathrm{L} \mathrm{KCL}, 1 \mathrm{mmol} / \mathrm{L} \mathrm{KH_{2 }} \mathrm{PO}_{4}, 0.1 \mathrm{mmol} / \mathrm{L}$ EGTA, $0.2 \%$ bovine serum albumin, and $50 \mathrm{mmol} / \mathrm{L}$ Tris-HCl; $\mathrm{pH} 7.4$ ) containing $0.1 \mathrm{mg} / \mathrm{mL}$ proteinase (Sigma-Aldrich, St. Louis, MO). Tissues were gently homogenized with six strokes using a motor-driven Teflon pestle in a glass chamber.

Homogenates were centrifuged at $700 \mathrm{~g}$ for $10 \mathrm{~min}$. Supernatants were centrifuged at $10,000 \mathrm{~g}$ for $10 \mathrm{~min}$, and pellets were washed and centrifuged at $7000 \mathrm{~g}$ for 3 min. The final pellets were suspended in suspension buffer containing $225 \mathrm{mmol} / \mathrm{L}$ mannitol, $75 \mathrm{mmol} / \mathrm{L} \mathrm{su}$ crose, $10 \mathrm{mmol} / \mathrm{L}$ Tris, and $0.1 \mathrm{mmol} / \mathrm{L}$ EDTA, pH 7.4. Finally, mitochondrial protein concentrations were measured by the bicinchoninic acid assay. These samples were used to measure mitochondrial function, for immunoblotting (SDS-PAGE and BN-PAGE), and lipidome analysis.
Cell culture and knockdown of CRLS1

H9c2 (ATCC ${ }^{\circledast}$ CRL-1446 ${ }^{\mathrm{TM}}$ ) cells were maintained in Dulbecco's Modified Eagle's Medium (4.5 g glucose/L; Sigma) containing 10\% fetal bovine serum (Cell Culture Bioscience), $100 \mathrm{IU} / \mathrm{mL}$ penicillin, and $100 \mathrm{IU} / \mathrm{mL}$ streptomycin in a humidified incubator (Sanyo, Osaka, Japan) at $37^{\circ} \mathrm{C}$ with $5 \% \mathrm{CO}_{2}$ in air. $\mathrm{H} 9 \mathrm{c} 2$ cells were plated in a T75 flask at a density of $1.0-1.6 \times 10^{6}$ cells and then transfected $24 \mathrm{~h}$ later with 25 or $50 \mathrm{nmol}$ of a nontargeting pool of small interfering RNA (siRNA) or siRNA against cardiolipin synthase 1 (CRLS1, Dharmacon, Brébières, France) using DharmaFECT reagent (Dharmacon). At 72-96h after transfection, the cells were harvested and lysed with homogenization buffer containing $10 \mathrm{mM}$ HEPES-KOH (pH 7.4), $220 \mathrm{mM}$ mannitol, and $0.07 \mathrm{M}$ sucrose with several deacetylase inhibitors. The mitochondrial fraction was also isolated by the sequential centrifugation method [19].

\section{Immunoblotting}

Immunoblotting was performed as described previously [20]. Briefly, mitochondrial protein samples were separated by SDS-PAGE and transferred to a polyvinylidene fluoride (PVDF) membrane (Bio-Rad, Hercules, CA). The

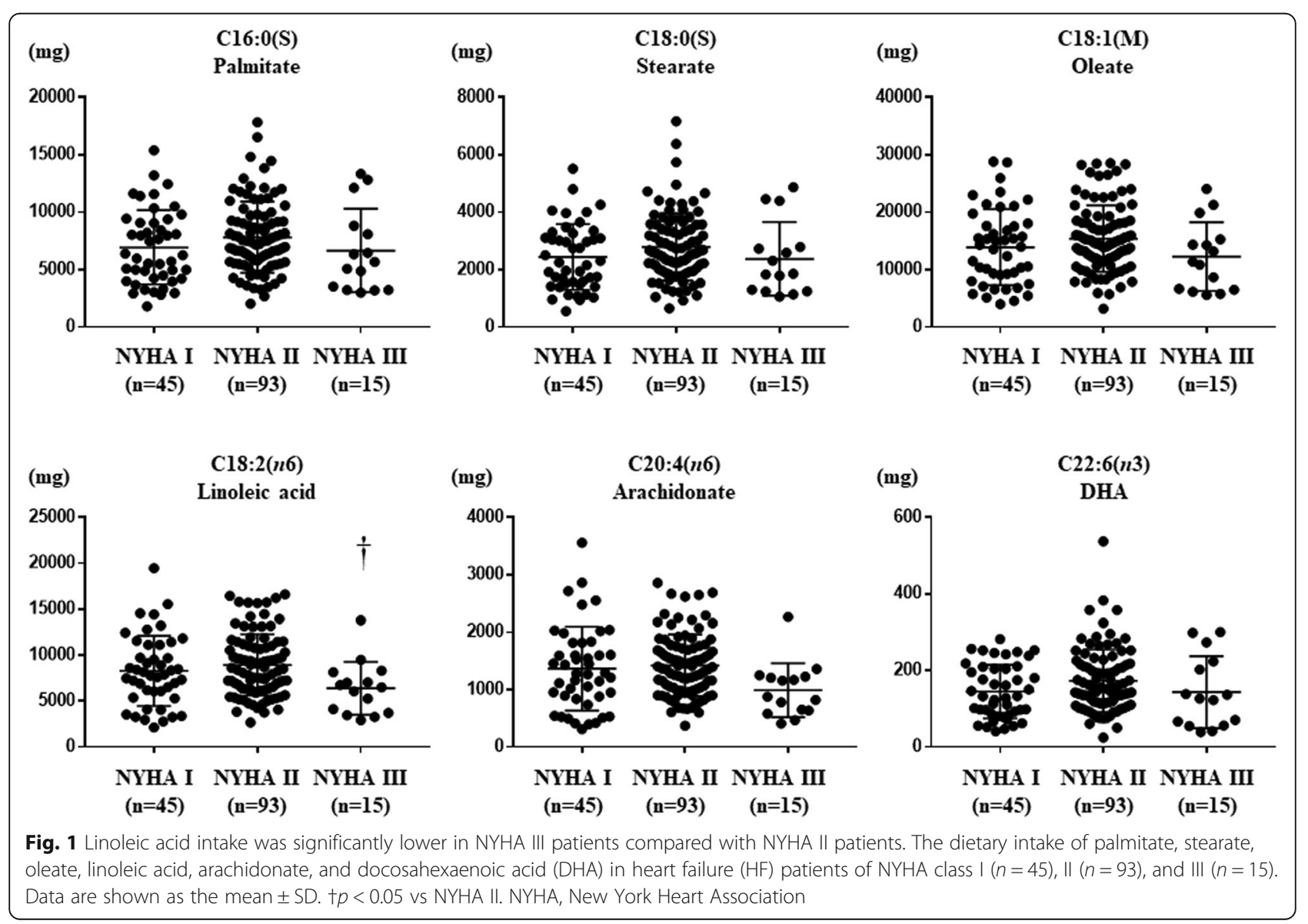


membrane was blocked for $1 \mathrm{~h}$ at room temperature in Tris-buffered saline containing $0.1 \%$ Tween 20 (TBS-T) buffer containing $3 \%$ milk, and then incubated with the primary antibodies (CRLS1 and total OXPHOS antibody cocktail; Abcam, Cambridge, MA) at a dilution of 1:1000 overnight at $4{ }^{\circ} \mathrm{C}$. After three washes with TBS-T, the membrane was incubated with a horseradish peroxideconjugated secondary antibody at a dilution of 1:5000 for 1 $\mathrm{h}$ at room temperature. After washing, the membrane was developed with ECL, ECL Prime, or SuperSignal West Dura Reagent (GE Healthcare Life Sciences, Piscataway, NJ) and then processed for detection with ChemiDoc XRS+ (Bio$\mathrm{Rad})$. The density of the band signals was quantified with Image J software (U.S. National Institutes of Health, Bethesda, MD). The expression levels of proteins are shown as values corrected with total protein (Coomassie brilliant blue [CBB] staining) of mitochondrial samples.

\section{BN-page}

BN-PAGE was performed as described previously [21]. Briefly, mitochondrial proteins were extracted with $5 \%$ digitonin (Invitrogen, Carlsbad, CA; protein:detergent ratio of $1: 10$ ) and $4 \times$ buffer (Invitrogen) on ice for 30 min. After centrifugation at $10,000 \mathrm{~g}$ for $10 \mathrm{~min}$ at $4{ }^{\circ} \mathrm{C}$, the supernatants were collected. The remaining lysates were combined with Coomassie blue G-250 dye (Invitrogen; protein:detergent ratio of 1:10) and added to $3-12 \%$ NativePAGE Novex Bis-Tris Gel (Invitrogen), then separated by electrophoresis using Anode and Cathode buffer (Invitrogen) at $10 \mathrm{~mA}$ for $1 \mathrm{~h}$ and at $150 \mathrm{~V}$ for $2 \mathrm{~h}$ on ice. Protein complexes in the samples after electrophoresis were denatured using denaturing buffer $(20 \mathrm{mmol}$ Tris, $200 \mathrm{mmol}$ glycine, and 1\% SDS). The gels were then transferred by electroblotting to PVDF membranes (Bio-Rad) using transfer buffer at $25 \mathrm{~V}$ for $2 \mathrm{~h}$.

\section{Mitochondria from mouse heart}
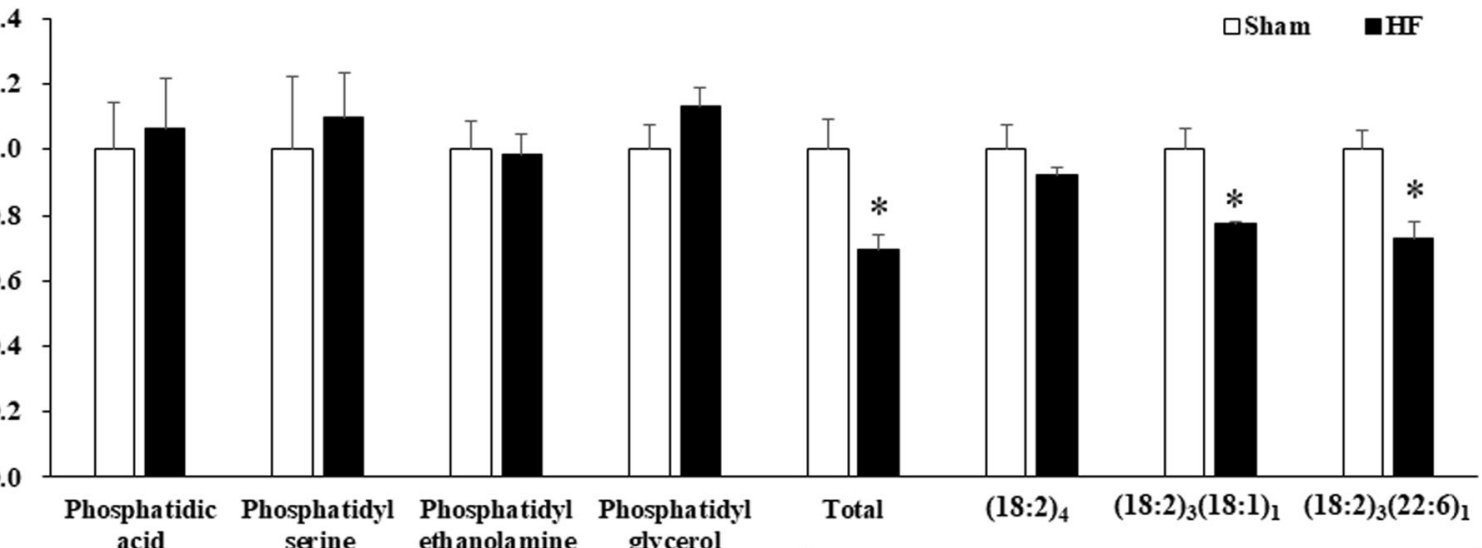

B

Complex I respiration

Complex I+II respiration

Complex II respiration
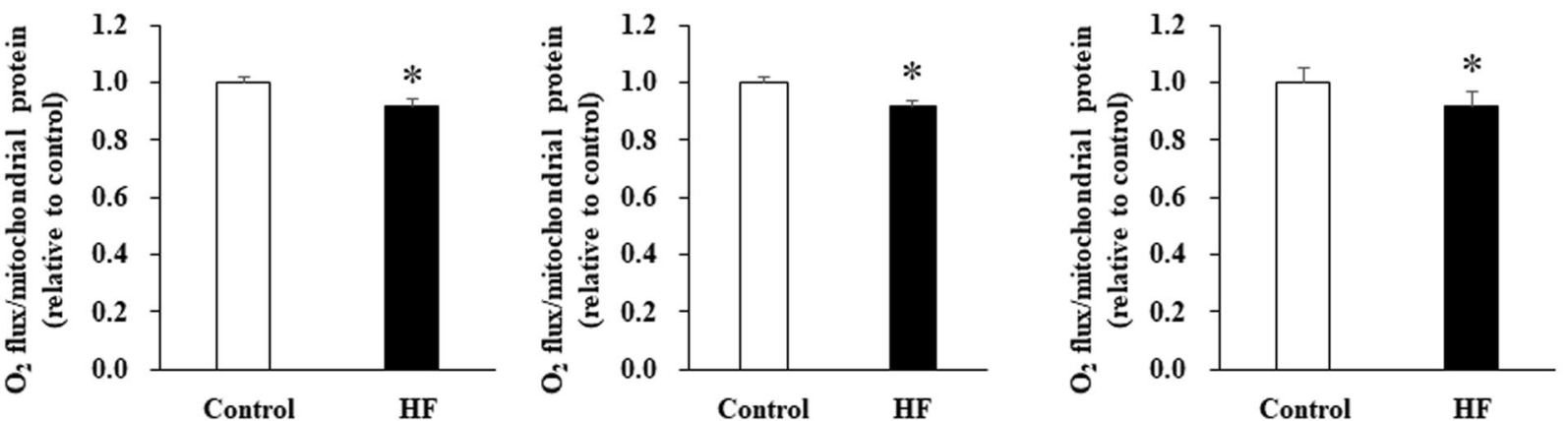

Fig. 2 Cardiolipin content and mitochondrial respiration were decreased in HF mice. a. Comparison of phospholipid fatty acids (phosphatidic acid, phosphatidyl serine, phosphatidyl ethanolamine, and phosphatidyl glycerol) and cardiolipin (total, $\left.(18: 2)_{4},(18: 2)_{3}(18: 1)_{1},(18: 2)_{3}(22: 6)_{1}\right)$ content in isolated heart mitochondria between control $(n=4)$ and HF mice $(n=4)$. b. Complex I-linked respiration, complex I+II-linked respiration, and complex II-linked respiration in heart mitochondria per mitochondrial protein in control $(n=4)$ and HF mice $(n=4)$. Data are shown as the mean \pm SE. ${ }^{*} p<0.05$ vs control mice 


\section{Measurement of mitochondrial OXPHOS capacity}

Mitochondrial respiratory capacities were measured in isolated mitochondria at $37{ }^{\circ} \mathrm{C}$ with a high-resolution respirometer (Oxygraph-2 k; Oroboros Instruments, Innsbruck, Austria) [21-23]. The respirometer chamber was filled with $2 \mathrm{~mL}$ of MiR05 medium, and then isolated mitochondria (approx. 30-100 $\mu \mathrm{g}$ ) were added, followed by the substrate ADP, and inhibitors in the following order: malate $(2 \mathrm{mmol} / \mathrm{L})$, pyruvate $(5 \mathrm{mmol} /$ $\mathrm{L})$, glutamate $10 \mathrm{mmol} / \mathrm{L}, \mathrm{MgCl}_{2}(3 \mathrm{mmol} / \mathrm{L})$, ADP $(10$ $\mathrm{mmol} / \mathrm{L}$; to measure $\mathrm{CI}$ respiration), succinate (10 $\mathrm{mmol} / \mathrm{L}$; to measure $\mathrm{CI}+\mathrm{II}$ respiration), and rotenone ( $0.5 \mu \mathrm{mol} / \mathrm{L}$; to measure complex II respiration). $\mathrm{O}_{2}$ consumption rates (i.e., respiratory rates) are expressed as the $\mathrm{O}_{2}$ flux normalized to the mitochondrial protein concentration $(\mu \mathrm{g} / \mu \mathrm{L})$. DatLab software (Oroboros Instruments) was used for data acquisition and data analysis.

Lipidome (phospholipid fatty acids, including cardiolipin) analysis by liquid chromatography-mass spectrometry (LC-MS)

To perform lipidome analysis, we analyzed $5 \mu \mathrm{L}$ of the supernatant by LC-MS using Nexera X2 (Shimadzu Corp., Kyoto, Japan) coupled with a Q-Exactive mass spectrometer (Thermo Fisher Scientific, San Jose, CA), followed by separation by reverse-phase LC using an Accucore C18 column $(150 \times 2.1 \mathrm{~mm}, 2.6 \mu \mathrm{m}$, Thermo Fisher Scientific). The mobile phase consisted of $\mathrm{H}_{2} \mathrm{O}$ : acetonitrile $(60: 40, \mathrm{v} / \mathrm{v})$ in $10 \mathrm{mM}$ ammonium formate and $0.1 \%$ formic acid (A) and isopropanol:acetonitrile $(90: 10, \mathrm{v} / \mathrm{v})$ in $10 \mathrm{mM}$ ammonium formate and $0.1 \%$ formic acid (B). The column oven temperature was $40^{\circ} \mathrm{C}$. The gradient elution program was as follows: a flow rate of $0.3 \mathrm{~mL} / \mathrm{min}$ : $0-1.5 \mathrm{~min}, 32 \% \mathrm{~B} ; 1.5-6 \mathrm{~min}, 32-58 \% \mathrm{~B}$; 6-18 min, $58-75 \%$ B; $18-21 \mathrm{~min}, 75-97 \%$ B; $21-25 \mathrm{~min}$, $97 \%$ B; $25.1-35 \mathrm{~min}, 32 \% \mathrm{~B}$. The parameters for the heated electrospray ionization source in negative-ion mode were as follows: flow rates of the sheath gas and auxiliary gas were 40 and 10 (arbitrary units), respectively. The spray voltage, capillary temperature, and heater temperature were maintained at $2.5 \mathrm{kV}, 350^{\circ} \mathrm{C}$, and $300^{\circ} \mathrm{C}$, respectively. The S-lens RF level was set at 50. The Q-Exactive mass spectrometer was operated at a resolving power of 70,000 in full-scan mode (scan range: $200-2000 \mathrm{~m} / \mathrm{z}$, automatic gain control target: 1e6); maximum ion injection time: $250 \mathrm{msec}$. Data processing was performed using LipidSearch software (Mitsui Knowledge Industry, Osaka, Japan), and ions corresponding to cardiolipin were subjected to statistical analysis.

Mitochondria from cardiomyocyte cell line

$\mathbf{A}$

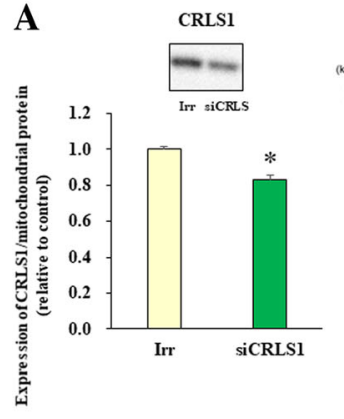

C

Complex I respiration

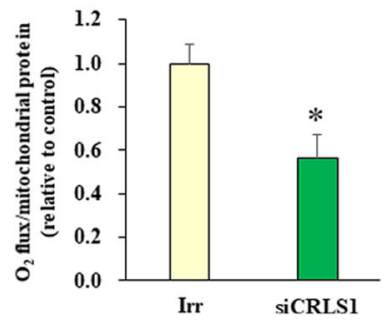

B Cardiolipin

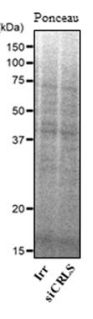

Complex I+II respiration

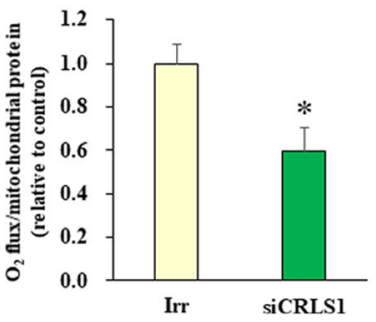

Complex II respiration

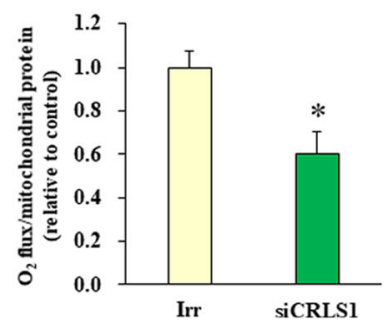

Fig. 3 Cardiolipin content and mitochondrial respiration were decreased in cardiomyocytes by siRNA-mediated blockade of cardiolipin synthase 1 (CRLS1). Assays were started at $72 \mathrm{~h}$ after the transfection. $\mathbf{a}$. Amount of CRLS1 in isolated mitochondria from cardiomyocytes treated with irrelevant SiRNA (Irr) $(n=6)$ or siCRLS1 $(n=6)$ (left). Total protein levels shown by Ponceau staining were used as the internal controls (right). The sizes of the molecular weight markers are indicated on the left in kDa. b. Cardiolipin content in isolated mitochondria from cardiomyocytes treated with an irrelevant siRNA (Irr, $n=5)$ or siCRLS1 $(n=5)$. C. Complex I-linked respiration, complex I+ II-linked respiration, and complex II-linked respiration in isolated mitochondria from cardiomyocytes treated with control $(n=4)$ and $\operatorname{siCRLS1}(n=4)$. Data are shown as the mean \pm SE. ${ }^{*} p<0.05$ vs Irr 


\section{Statistical analysis}

Data of the dietary intake of fatty acids, demographic factors, causes of heart failure, medications, and echocardiographic parameters in HF patients were summarized as the mean \pm standard deviation (SD). One-way ANOVA was used to compare continuous variables. Categorical variables are presented as numbers or percentages, and were compared by the chi-squared test. All analyses were performed using JMP Pro 14.0 (SAS Institute, Cary, $\mathrm{NC}$,). Other data were expressed as the mean \pm standard error of the mean (SE). Statistical analyses were performed using the Student $t$-test for comparisons between two groups and one-way analysis of variance followed by the Tukey test for three groups with GraphPad Prism 6 software (GraphPad, San Diego, CA). A univariate linear regression model was used to determine the correlation between two variables. A $P$-value less than 0.05 was considered to indicate a statistically significant difference between two groups.

\section{Results}

Linoleic acid intake reduces in $\mathrm{HF}$ patients

We first compared the dietary intake of each fatty acid among HF patients of NYHA class I $(n=45)$, II $(n=96)$, and III $(n=15)$. Table 1 shows demographic factors, causes of heart failure, medications, and echocardiographic parameters in each NYHA class. Rate of men among all patients and the use of an ACE inhibitor or ARB as medication were significantly different among the three groups (Table 1). On the other hand, there were no differences in other data, including the cause of heart failure and cardiac function (Table 1). Among the several fatty acids that we analyzed, the dietary intake of linoleic acid was significantly lower in NYHA III patients than in NYHA II patients (Fig. 1). A previous study demonstrated that cardiolipin content was decreased in the cardiac mitochondria of aged spontaneous hypertensive HF rats, and linoleic acid is the major fatty acid moiety of cardiolipin, as mentioned earlier [6]. We were thus interested in further studying the possible association between the dietary intake of linoleic acid and the cardiolipin content of cardiac mitochondria, particularly with regard to cardiac mitochondrial function.

\section{Heart mitochondrial cardiolipin and function reduce in $\mathrm{HF}$ mice}

Consistent with a previous report [6], we then found that levels of phospholipid fatty acids (phosphatidic acid, phosphatidyl serine, phosphatidyl ethanolamine, and phosphatidyl glycerol) do not differ between a mouse model of HF after myocardial infarction and control mice, whereas the total cardiolipin content,
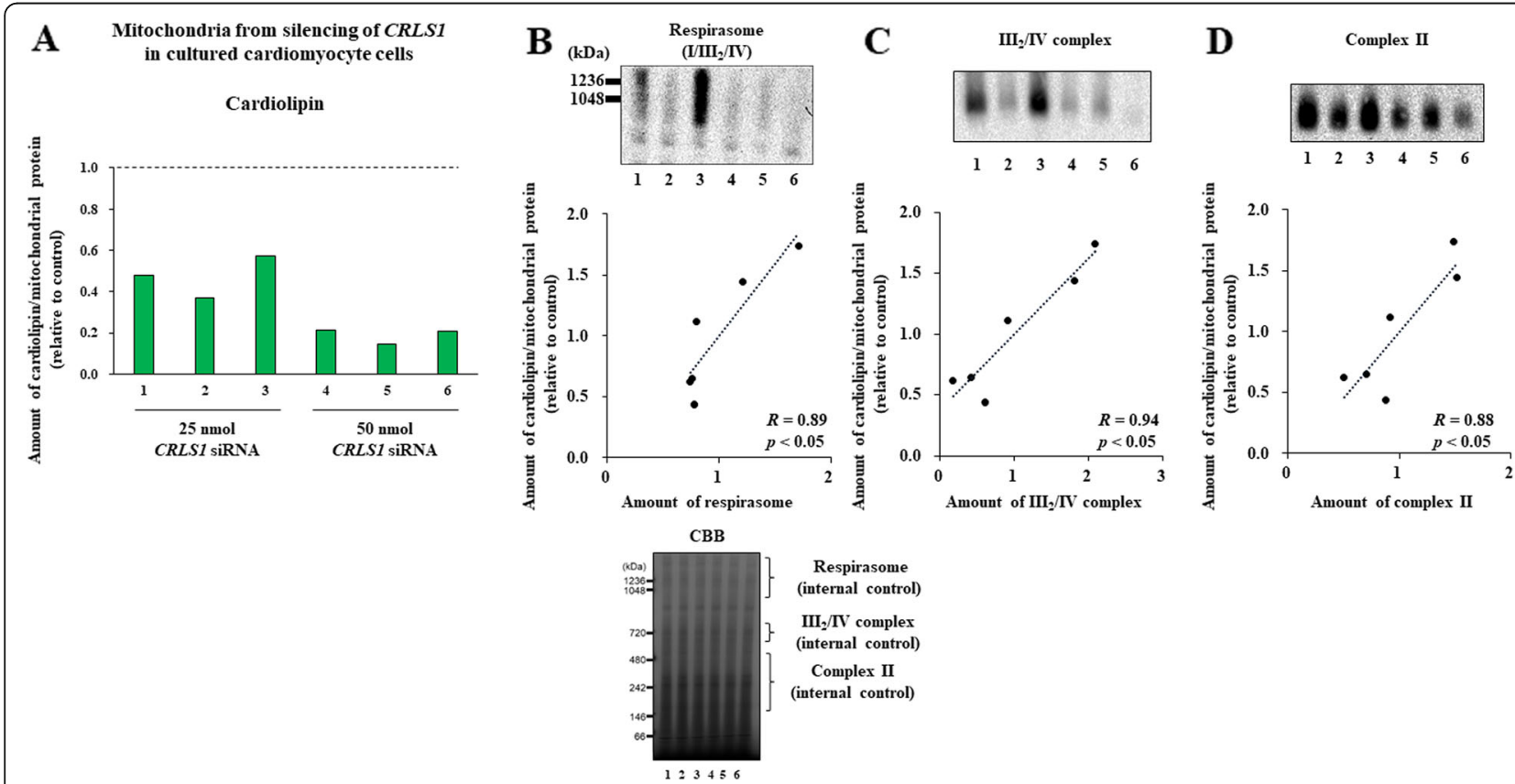

Fig. 4 Amount of supercomplexes and complex II significantly correlated with cardiolipin content in mitochondria isolated from siCRLS1-treated cardiomyocytes. Assays were started at $96 \mathrm{~h}$ after the transfection with 25 or $50 \mathrm{nmol}$ siRNA ( $n=3$ each). a. The cardiolipin content in mitochondria isolated from siCRLS1 cardiomyocytes are shown relative to Irr $(n=6)$. b. Correlation between cardiolipin content and the amount of respirasomes $\left(I / I I_{2} / I_{n}\right)$. c. Correlation between cardiolipin content and the amount of complex $\|_{2} / \mathrm{IV}$. $\mathbf{d}$. Correlation between cardiolipin content and the amount of complex II. Pearson correlation coefficients (R) and $p$-values are shown 
including the molecular species composition of cardiolipin $\left[(18: 2)_{3}(18: 1)_{1}\right.$ and $\left.(18: 2)_{3}(22: 6)_{1}\right]$ in cardiac mitochondria, is significantly lower in HF mice [1214] than in control mice (Fig. 2a). In cardiac mitochondria of these HF mice, both CI-linked respiration $(\mathrm{CI}+\mathrm{III}+\mathrm{IV})$ and complex II-linked respiration (CII + III + IV) per mitochondrial protein, as well as CI + IIlinked respiration were significantly reduced compared with control mice (Fig. 2b).
Similar to our earlier studies, we herein confirmed using these HF mice that cardiac function (\%fraction shortening), as analyzed by echocardiography, was significantly decreased and that the LV was larger than control mice at 4 weeks after surgery (control [ $n=4]$ vs HF $[n=5]: \% \mathrm{FS}(\%), 45.5 \pm 0.9$ vs $12.9 \pm 3.4, p<0.05$; LV end-diastolic diameter, $(\mathrm{mm}), 3.25 \pm 0.14$ vs $5.04 \pm 0.26$, $p<0.05$; LV end-systolic diameter $(\mathrm{mm}), 1.78 \pm 0.10$ vs $4.42 \pm 0.39, p<0.05)$.

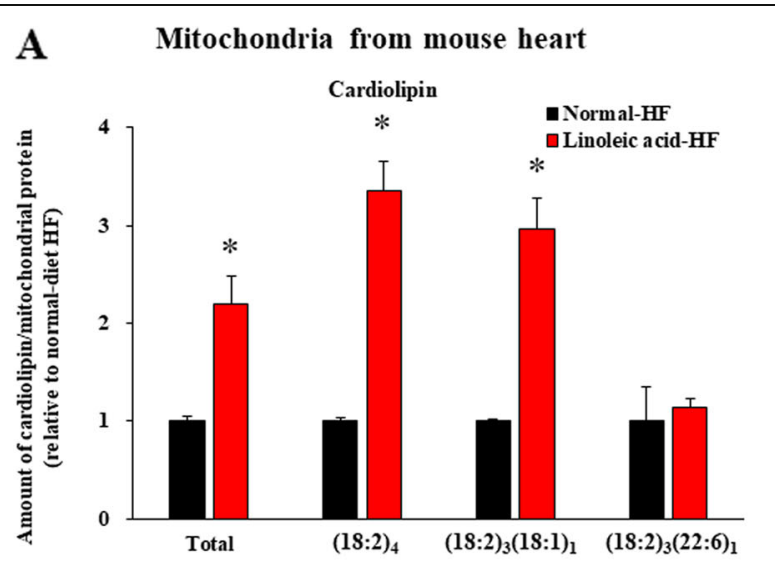

B

Complex I respiration

Complex I+II respiration

Complex II respiration
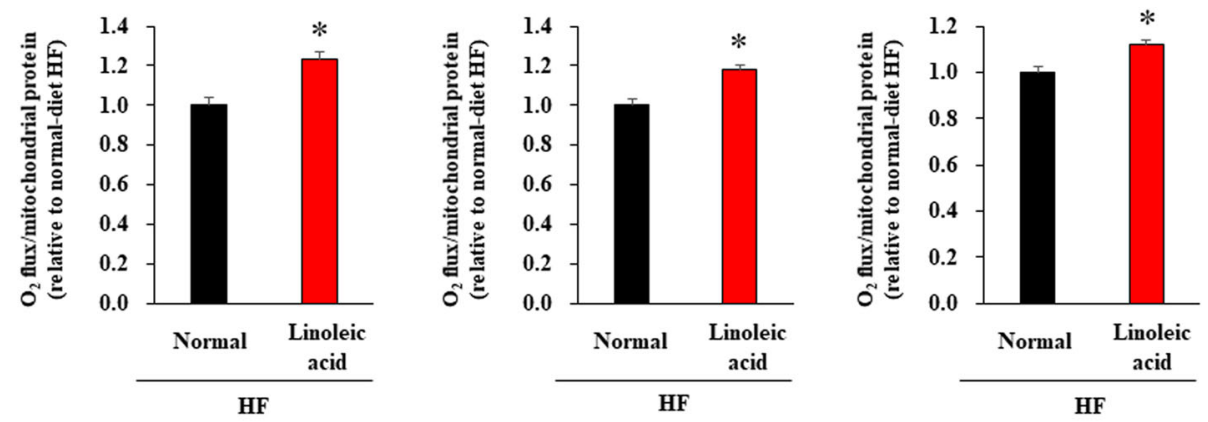

C

LV end-diastolic diameter

LV end-systolic diameter
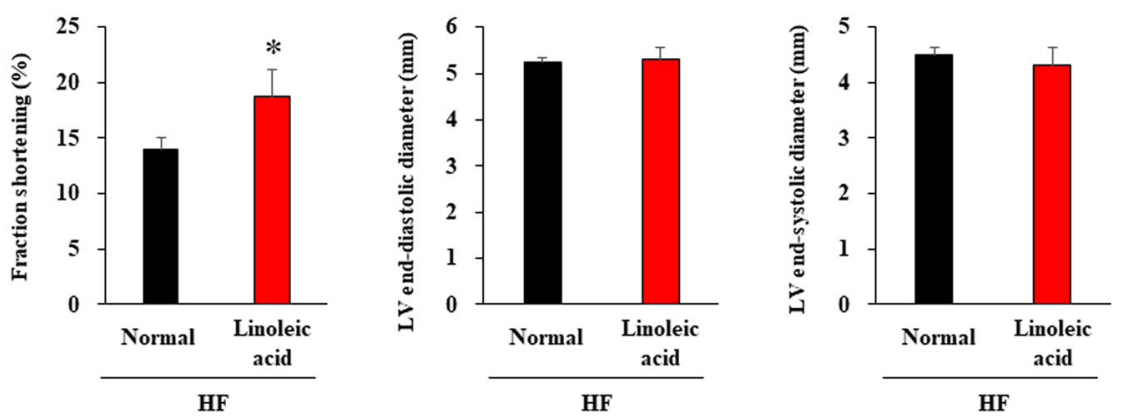

Fig. 5 Supplementation of linoleic acid to HF mice increased cardiolipin content and improved mitochondrial respiration and cardiac function. a. Cardiolipin contents (total, $\left.(18: 2)_{4},(18: 2)_{3}(18: 1)_{1},(18: 2)_{3}(22: 6)_{1}\right)$ in isolated heart mitochondria from HF mice fed a normal diet $(n=5)$ and HF mice fed a linoleic acid-rich diet $(n=5)$. b. Complex I-linked respiration, complex I + I-linked respiration, and complex $\|$-linked respiration per heart mitochondrial protein in HF mice $(n=5)$ and HF mice fed a linoleic acid-rich diet $(n=5)$. c. LV function, LV end-diastolic diameter, and LV endsystolic diameter in HF mice fed a normal diet $(n=25)$ and HF mice fed a linoleic acid-rich diet $(n=8)$. Data are shown as the mean \pm SE. LV, left ventricle. * $p<0.05$ vs HF mice on a normal diet" 
Assembly of supercomplexes and complex II correlates with cardiolipin content in mitochondria isolated from siCRLS1-treated cardiomyocytes

The above results suggested a possible link between the reduced amounts of cardiolipin and the reduced assembly of the mitochondrial OXPHOS components in cardiomyocytes. We then confirmed this association using cultured rat $\mathrm{H} 9 \mathrm{c} 2$ cardiomyocytes [24]. Silencing of CRLS1, which encodes cardiolipin synthesis 1 , by the siRNA method significantly reduced cardiolipin levels in these cells (Fig. 3a,b). In siCRLS1 cells, CI-linked, CIIlinked, as well as CI + II-linked mitochondrial respiration were significantly reduced compared with irrelevant siRNA-treated control cells (Fig. 3c). We next varied the concentration of siRNA ( 25 or $50 \mathrm{nmol}$ ), which resulted in differences in CRLS1 expression levels and cardiolipin concentrations (see Fig. 4a). Taking advantage of such variations, we then analyzed the association between cardiolipin content and the assembly of OXPHOS components. We found significant correlations between cardiolipin content per mitochondrial protein and the amounts of the $\mathrm{CI} / \mathrm{III}_{2} / \mathrm{IV}$ respirasome and $\mathrm{CIII}_{2} / \mathrm{IV}$ supercomplex, as well as CII-subunit assembly in mitochondria isolated from these cells (Fig. 4b-d). Therefore, these results collectively indicated that a reduction in cardiolipin levels is closely associated with reduced mitochondrial respiration in cardiomyocytes, as has been implicated in various types of cells and organisms as mentioned above.

\section{Supplementation of linoleic acid improves heart mitochondrial crisis and cardiac dysfunction in HF mice} We then sought to investigate the associations among the dietary intake of linoleic acid, cardiolipin levels in cardiac mitochondria, and $\mathrm{CI} / \mathrm{CII}$-linked respiration of cardiomyocytes in vivo. We fed HF model mice with a diet supplemented with a high amount of linoleic acid (about $150 \mu \mathrm{g} /$ day), and measured cardiolipin content and OXPHOS activities in their heart mitochondria after 4 weeks on a continued linoleic acid diet. Total cardiolipin content, including the molecular species composition of cardiolipin $\left[(18: 2)_{4}\right.$ and $\left.(18: 2)_{3}(18: 1)_{1}\right]$, in cardiac mitochondria were significantly improved by linoleic acid supplementation, to levels almost equal to those of healthy mice (Fig. 5a and Fig. 2a). This supplementation also improved both complex I-linked and complex IIlinked respiration of cardiac mitochondria (Fig. 5b) and LV function (Fig. 5c)." On the other hand, contrary to cultured cardiomyocytes, we did not observe any notable improvement in $\mathrm{CI} / \mathrm{III}_{2} / \mathrm{IV}$ respirasome supercomplex

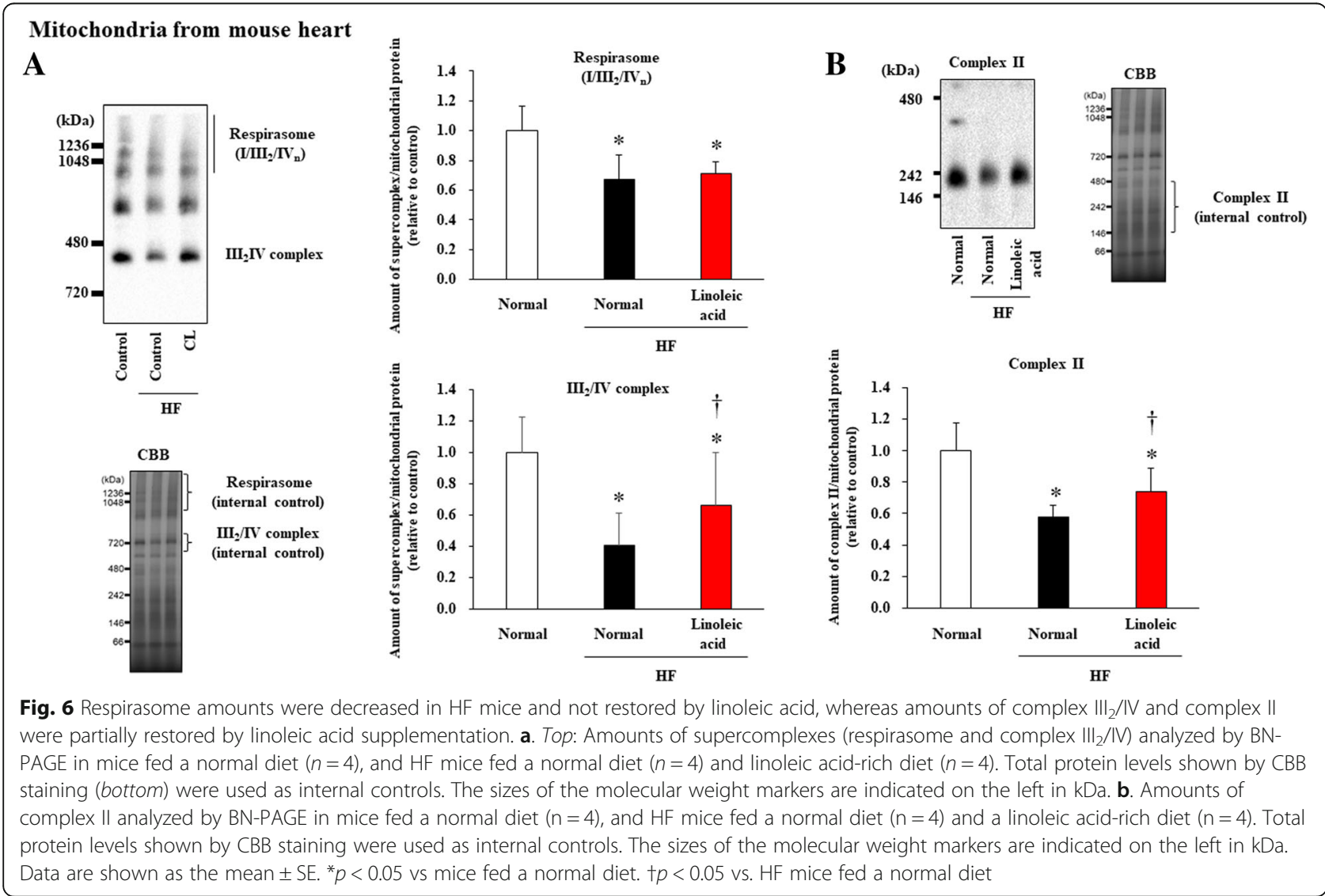


formation in these HF mice fed linoleic acid, whereas assembly of the CIII2/IV supercomplex and CII subunits were indeed significantly improved (Fig. 6a,b).

\section{Conclusions}

Linoleic acid is an essential omega- 6 fatty acid, and a prospective study demonstrated that a high conjugated linoleic acid concentration is associated with a lower risk of HF incidence in older men [25], whereas its excess intake is well known to be harmful to human health, including the increased risk of arteriosclerosis [26]. Ironically, however, we here found that HF patients who take less amounts of linoleic acid in their daily diets have more severe HF. Furthermore, we demonstrated that both CI-linked and CII-linked respiration of heart mitochondria are affected by HF, and that dietary intake of linoleic acid improves both of these respiratory mechanisms, primarily through an improvement in the assembly of CII subunits and the $\mathrm{CIII}_{2}$ /CIV supercomplex.

In our animal experiments, dietary intake of linoleic acid did not improve the assembly of the $\mathrm{CI} / \mathrm{III}_{2} / \mathrm{IV}$ respirasome, whereas in cultured cells, cardiolipin content was statistically associated with the assembly of the $\mathrm{CI} / \mathrm{III}_{2} / \mathrm{IV}$ respirasome, CII-subunits, and the $\mathrm{CIII}_{2} / \mathrm{CIV}$ supercomplex. Therefore, it is likely that dysregulation of respirasome assembly that occurs in HF cannot be easily repaired by the enhanced intake of linoleic acid or by enhanced cardiolipin content. We have no clear explanation for this, but it is possible that this phenomenon is simply a reflection of the possible ordered assembly of OXPHOS components. Nevertheless, whether other factors or events that are involved in respirasome assembly, or other aspects of mitochondrial function, are specifically affected by HF should be further investigated. The mechanisms by which cardiolipin content are reduced by HF, and whether cardiolipin content can be restored to a certain extent in HF mice by linoleic acid supplementation, also needs to be clarified.

We hence concluded that dietary supplementation of linoleic acid is beneficial for improving cardiac malfunction in HF, but is unable to completely cure HF.

\section{Abbreviations}

BDHQ: brief self-administered diet history questionnaire; BN-PAGE: Blue Native-Polyacrylamide Gel Electrophoresis; CBB: Coomassie Brilliant Blue; Cl: complex I; Cll: complex II; CRLS1: cardiolipin synthase 1; HF: heart failure; LA: linoleic acid; LV: left ventricle; NYHA: New York Heart Association; OXPHOS: oxidative phosphorylation

\section{Acknowledgments}

The authors thank Yuki Kimura and Miwako Yamane for their technical assistance, and Ayae Oda, Misaki Kihara, Naoko Toshiro, Tsukusu Yamanaka, and Misato Kobayashi for secretarial support, and H.A. Popiel for her critical reading of the manuscript.

\section{Disclosures}

The authors state that there are no disclosures to provide.

\section{Authors' contributions}

SM and ST designed the study. SM, ST, HM, TF, NK, DS, YU, and DK performed the experiments and SM, ST, HM, TF, NK, DS, YU, DK, HS, and SK analyzed the data. SM, ST, HS, and SK wrote the manuscript. All authors read and approved the final version of the manuscript.

\section{Funding}

This work was supported in part by grants from Japanese Grant-In-Aid for Scientific Research (JP17H04758 [to S.T.], and 18H03187 [to S.K.]), Grant-in-Aid for Challenging Exploratory Research (19 K22791 [to S.T.], the Japan Foundation for Applied Enzymology (to S.T.), a Hokkaido Heart Association Grant for Research (to S.T.), the MSD Life Science Foundation (to S.T.), the Uehara Memorial Foundation (to S.T.), the Cardiovascular Research Fund, Tokyo, Japan (to S.T.), the Fukuda Memorial Foundation for Medical Research (to S.T.), a Kimura Memorial Heart Foundation Research Grant for 2017 (to S.T.), the SENSHIN Medical Research Foundation (to S.T.), the Nakatomi Foundation (to S.T.), the Japan Heart Foundation (to S.T.), a Sasakawa Scientific Research Grant from The Japan Science Society (to S.T.), and the Center of Innovation Program from the Japan Science and Technology Agency.

\section{Availability of data and materials}

The datasets generated during and/or analyzed during the current study are available from the corresponding author upon reasonable request.

Ethics approval and consent to participate

All procedures and animal care were approved by our institutional animal research committee and conformed to the Animal Care Guidelines for the Care and Use of Laboratory Animals at Hokkaido University Graduate School of Medicine. The procedures and care were also in accordance with relevant national and international guidelines and the Guide for the Care and Use of Laboratory Animals published by the U.S. National Institutes of Health. The study was approved by the Medical Ethics Committee of Hokkaido University Hospital in accordance with the ethical principles described in the

Declaration of Helsinki (2013 revised version), and written informed consent was obtained from all participating subjects.

\section{Consent for publication}

Not applicable.

\section{Competing interests}

The authors declare that they have no conflicts of interest associated with this manuscript.

\section{Author details}

${ }^{1}$ Department of Cardiovascular Medicine, Hokkaido University Graduate School of Medicine, Kita-15, Nishi-7, Kita-ku, Sapporo 060-8638, Japan. ${ }^{2}$ Department of Molecular Biology, Hokkaido University Graduate School of Medicine, Sapporo, Japan. ${ }^{3}$ Faculty of Lifelong Sport, Department of Sports Education, Hokusho University, Ebetsu, Japan. ${ }^{4}$ Research Fellow of the Japan Society for the Promotion of Science, Tokyo, Japan. ${ }^{5}$ Clinical Laboratories, Kyushu University Hospital, Fukuoka, Japan. ${ }^{6}$ Department of Clinical Chemistry and Laboratory Medicine, Graduate School of Medical Sciences, Kyushu University, Fukuoka, Japan.

Received: 26 May 2019 Accepted: 24 September 2019

Published online: 16 October 2019

References

1. Bui AL, Horwich TB, Fonarow GC. Epidemiology and risk profile of heart failure. Nat Rev Cardiol. 2011;8:30-41.

2. Tran HA, Lin F, Greenberg BH. Potential new drug treatments for congestive heart failure. Expert Opin Investig Drugs. 2016;25:811-26.

3. Colonna P, Sorino M, D'Agostino C, Bovenzi F, De Luca L, Arrigo F. Nonpharmacologic care of heart failure: counseling, dietary restriction, rehabilitation, treatment of sleep apnea, and ultrafiltration. Am J Cardiol. 2003:91:41-50.

4. Abbott SK, Else PL, Atkins TA, Hulbert AJ. Fatty acid composition of membrane bilayers: importance of diet polyunsaturated fat balance. Biochim Biophys Acta. 1818;2012:1309-17. 
5. Ting HC, Chen LT, Chen JY, Huang YL, Xin RC, Chan JF, Hsu YH. Double bonds of unsaturated fatty acids differentially regulate mitochondrial cardiolipin remodeling. Lipids Health Dis. 2019;18:53.

6. Mulligan CM, Sparagna GC, Le CH, De Mooy AB, Routh MA, Holmes MG, Hickson-Bick DL, Zarini S, Murphy RC, Xu FY, et al. Dietary linoleate preserves cardiolipin and attenuates mitochondrial dysfunction in the failing rat heart. Cardiovasc Res. 2012;94:460-8.

7. Fiedorczuk K, Letts JA, Degliesposti G, Kaszuba K, Skehel M, Sazanov LA. Atomic structure of the entire mammalian mitochondrial complex I. Nature. 2016:538:406-10.

8. Schwall CT, Greenwood VL, Alder NN. The stability and activity of respiratory complex II is cardiolipin-dependent. Biochim Biophys Acta. 1817;2012:1588-96.

9. Mileykovskaya E, Dowhan W. Cardiolipin-dependent formation of mitochondrial respiratory supercomplexes. Chem Phys Lipids. 2014;179:42-8.

10. Bazan S, Mileykovskaya E, Mallampalli VK, Heacock P, Sparagna GC, Dowhan W. Cardiolipin-dependent reconstitution of respiratory supercomplexes from purified Saccharomyces cerevisiae complexes III and IV. J Biol Chem. 2013; 288:401-11

11. Takayama M, Arai Y, Sasaki S, Hashimoto M, Shimizu K, Abe Y, Hirose N. Association of marine-origin n-3 polyunsaturated fatty acids consumption and functional mobility in the community-dwelling oldest old. J Nutr Health Aging. 2013;17:82-9.

12. Fukushima A, Kinugawa S, Takada S, Matsushima S, Sobirin MA, Ono T, Takahashi M, Suga T, Homma T, Masaki Y, et al. (pro) renin receptor in skeletal muscle is involved in the development of insulin resistance associated with postinfarct heart failure in mice. Am J Physiol Endocrinol Metab. 2014:307:E503-14.

13. Kinugawa S, Tsutsui H, Hayashidani S, Ide T, Suematsu N, Satoh S, Utsumi H, Takeshita A. Treatment with dimethylthiourea prevents left ventricular remodeling and failure after experimental myocardial infarction in mice: role of oxidative stress. Circ Res. 2000;87:392-8.

14. Iwamoto M, Kono M, Kawamoto D, Tomoyori H, Sato M, Imaizumi K Differential effect of walnut oil and safflower oil on the serum cholesterol level and lesion area in the aortic root of apolipoprotein E-deficient mice. Biosci Biotechnol Biochem. 2002;66:141-6.

15. Tsuda M, Fukushima A, Matsumoto J, Takada S, Kakutani N, Nambu H, Yamanashi K, Furihata T, Yokota T, Okita K, et al. Protein acetylation in skeletal muscle mitochondria is involved in impaired fatty acid oxidation and exercise intolerance in heart failure. J Cachexia Sarcopenia Muscle. 2018:9:844-59.

16. Tonkonogi M, Sahlin K. Rate of oxidative phosphorylation in isolated mitochondria from human skeletal muscle: effect of training status. Acta Physiol Scand. 1997;161:345-53.

17. Yokota T, Kinugawa S, Hirabayashi K, Matsushima S, Inoue N, Ohta Y, Hamaguchi S, Sobirin MA, Ono T, Suga T, et al. Oxidative stress in skeletal muscle impairs mitochondrial respiration and limits exercise capacity in type 2 diabetic mice. Am J Physiol Heart Circ Physiol. 2009;297:H1069-77.

18. Hey-Mogensen M, Gram M, Jensen MB, Lund MT, Hansen CN, ScheibyeKnudsen M, Bohr VA, Dela F. A novel method for determining human ex vivo submaximal skeletal muscle mitochondrial function. J Physiol. 2015; 593:3991-4010.

19. Frezza C, Cipolat S, Scorrano L. Organelle isolation: functional mitochondria from mouse liver, muscle and cultured fibroblasts. Nat Protoc. 2007:2:287-95.

20. Maekawa S, Mori D, Nishiya T, Takikawa O, Horinouchi T, Nishimoto A, Kajita E, Miwa S. OCTN2VT, a splice variant of OCTN2, does not transport carnitine because of the retention in the endoplasmic reticulum caused by insertion of 24 amino acids in the first extracellular loop of OCTN2. Biochim Biophys Acta. 1773:2007:1000-6.

21. Takada S, Masaki Y, Kinugawa S, Matsumoto J, Furihata T, Mizushima W, Kadoguchi T, Fukushima A, Homma T, Takahashi M, et al. Dipeptidyl peptidase-4 inhibitor improved exercise capacity and mitochondrial biogenesis in mice with heart failure via activation of glucagon-like peptide1 receptor signalling. Cardiovasc Res. 2016;111:338-47.

22. Christiansen LB, Dela F, Koch J, Hansen CN, Leifsson PS, Yokota T. Impaired cardiac mitochondrial oxidative phosphorylation and enhanced mitochondrial oxidative stress in feline hypertrophic cardiomyopathy. Am J Physiol Heart Circ Physiol. 2015;308:H1237-47.

23. Mizushima W, Takahashi H, Watanabe M, Kinugawa S, Matsushima S, Takada S, Yokota T, Furihata T, Matsumoto J, Tsuda M, et al. The novel heart-specific RING finger protein 207 is involved in energy metabolism in cardiomyocytes. J Mol Cell Cardiol. 2016;100:43-53.
24. Chou HC, Chen YW, Lee TR, Wu FS, Chan HT, Lyu PC, Timms JF, Chan HL. Proteomics study of oxidative stress and Src kinase inhibition in $\mathrm{H} 9 \mathrm{C2}$ cardiomyocytes: a cell model of heart ischemia-reperfusion injury and treatment. Free Radic Biol Med. 2010:49:96-108.

25. Wannamethee SG, Jefferis BJ, Lennon L, Papacosta O, Whincup PH, Hingorani AD. Serum conjugated linoleic acid and risk of incident heart failure in older men: the British regional heart study. J Am Heart Assoc. 2018;7.

26. Ramsden CE, Zamora D, Leelarthaepin B, Majchrzak-Hong SF, Faurot KR, Suchindran CM, Ringel A, Davis JM, Hibbeln JR. Use of dietary linoleic acid for secondary prevention of coronary heart disease and death: evaluation of recovered data from the Sydney diet heart study and updated metaanalysis. BMJ. 2013;346:e8707.

\section{Publisher's Note}

Springer Nature remains neutral with regard to jurisdictional claims in published maps and institutional affiliations.

\section{Ready to submit your research? Choose BMC and benefit from:}

- fast, convenient online submission

- thorough peer review by experienced researchers in your field

- rapid publication on acceptance

- support for research data, including large and complex data types

- gold Open Access which fosters wider collaboration and increased citations

- maximum visibility for your research: over $100 \mathrm{M}$ website views per year

At BMC, research is always in progress.

Learn more biomedcentral.com/submissions 